\title{
Klares Bekenntnis für faire Rahmenbedingungen beim E-Lending in Bibliotheken
}

\section{Deutscher Bibliotheksverband begrüßt Koalitionsvertrag der Ampelkoalition}

https://doi.org/10.1515/bd-2022-0016

Der Deutsche Bibliotheksverband e.V. (dbv) äußert sich zufrieden mit den Inhalten des am 24.11.2021 vorgestellten Koalitionsvertrages. Er fordert zugleich eine gesetzliche Regelung für den Verleih von E-Books durch Bibliotheken.

\section{E-Lending}

Der dbv begrüßt das klare Bekenntnis der kommenden Bundesregierung, faire Rahmenbedingungen beim E-Lending in Bibliotheken $\mathrm{zu}$ schaffen. Der dbv bekräftigt in diesem Zusammenhang seine Forderung nach einer gesetzlichen Regelung, damit Öffentliche Bibliotheken E-Book-Lizenzen gleich nach Erscheinen zu angemessenen Bedingungen käuflich erwerben können - bei gleichzeitiger fairer Vergütung der Autorinnen.

\section{Urheberrecht}

Der dbv begrüßt, dass sich die kommende Bundesregierung für ein wissenschaftsfreundlicheres Urheberrecht einsetzen will. Aus Sicht des dbv bedarf es weiterer zeitgemäßer Regelungen im Urheberrecht, damit Studierende, Lehrende und Forschende auch in Zukunft nachhaltig mit Informationen und Medien durch Hochschulbibliotheken versorgt werden können.

\section{Open Science}

Der dbv unterstützt die Bemühungen der kommenden Bundesregierung, Open Access und Open Science zu stärken. Open Access soll in der kommenden Legislaturperiode als gemeinsamer Standard etabliert werden. 


\section{Sonntagsöffnung}

Der dbv begrüßt, dass die kommende Bundesregierung die Sonntagsöffnungen für Öffentliche Bibliotheken bundesweit ermöglichen möchte. Das Land Nordrhein-Westfalen ist bislang das einzige Bundesland, in dem die Möglichkeit zur Sonntagsöffnung von Öffentlichen Bibliotheken gesetzlich geregelt ist.

\section{Förderung von Bibliotheken durch den Bund}

Der dbv begrüßt, dass die kommende Bundesregierung die „Neustart-Programme“ zunächst fortführen wird. Der dbv setzt im Rahmen von Neustart Kultur das Digitalprogramm „WissensWandel“ um, das Bibliotheken und Archive bei der Umsetzung ihrer digitalen Transformation unterstützt. Zur Stärkung des Zusammenhalts plant die Bundesregierung zudem die Aufstockung und Vereinfachung von Investitions- und Sanierungsprogrammen u. a. im Bereich Kultur z. B. für Bibliotheken.

\section{Nachhaltigkeit}

Der dbv begrüßt, dass die 17 globalen Nachhaltigkeitsziele der Vereinten Nationen (SDG) zur Richtschnur der kommenden Legislaturperiode werden sollen. Bibliotheken leisten als meistbesuchte, niederschwellig zugängliche Einrichtungen, in denen Wissen, Information und Infrastruktur geteilt und langfristig zur Verfügung gestellt wird, einen wichtigen Beitrag zur Erreichung der Nachhaltigkeitsziele. Der dbv fordert, dass eine Weiterentwicklung der Deutschen Nachhaltigkeitsstrategie, wie sie im Koalitionsvertrag vorgesehen ist, unter Einbindung von Bibliotheken stattfindet.

\section{Inklusive Bildung}

Der dbv begrüßt, dass die kommende Bundesregierung allen Menschen unabhängig von ihrer Herkunft beste Bildungschancen und eine inklusive Bildung bieten möchte und dafür auch die Einbindung außerschulischer Akteure unterstützt. Außerschulische Akteure wie z. B. Bibliotheken spielen im Bereich der Leseförderung und der Medienbildung eine zentrale Rolle und arbeiten dabei eng mit den formalen Bildungseinrichtungen zusammen.

Zum Koalitionsvertrag der kommenden Bundesregierung sagt der Bundesvorsitzende des dbv, Prof. Dr. Andreas Degkwitz: „Die Ampelkoalition hat wichtige Forderungen des dbv in den Koalitionsvertrag aufgenommen. Damit erkennen die Koalitionspartner den hohen gesellschaftlichen, bildungspolitischen und kulturellen Stellenwert an, den Bibliotheken für den Zusammenhalt unserer Gesellschaft haben. Der dbv begrüßt das klare Bekenntnis der kommenden Bundesregierung, sich für die Weiterentwicklung von zeitgemäßen Bibliotheksangeboten und den digitalen Wandel in Bibliotheken einzusetzen.“ 
Der Koalitionsvertrag kann hier abgerufen werden: https://www.spd.de/ fileadmin/Dokumente/Koalitionsvertrag/Koalitionsvertrag_2021-2025.pdf.

\section{Deutscher Bibliotheksverband}

Der Deutsche Bibliotheksverband e.V. (dbv) vertritt mit seinen fast 2.100 Mitgliedern bundesweit über 9.000 Bibliotheken mit 25.000 Beschäftigten und 11 Millionen Nutzer`innen. Sein zentrales Anliegen ist es, Bibliotheken zu stärken, damit sie allen Bürger ${ }^{\star}$ innen freien Zugang zu Informationen ermöglichen. Der Verband setzt sich ein für die Entwicklung innovativer Bibliotheksleistungen für Wissenschaft und Gesellschaft. Als politische Interessensvertretung unterstützt der dbv die Bibliotheken insbesondere auf den Feldern Informationskompetenz und Medienbildung, Leseförderung und bei der Ermöglichung kultureller und gesellschaftlicher Teilhabe für alle Bürger^innen.

URL: www.bibliotheksverband.de

\section{Pressekontakt}

Kristin Bäßler

Deutscher Bibliotheksverband

Leitung Kommunikation / Pressesprecherin

Tel.: + 49 (0)30 6449899 25 | E-Mail: baessler@bibliotheksverband.de 